\title{
Joint probability discrimination between stationary tissue and blood velocity signals
}

\section{Schlaikjer, Malene; Jensen, Jørgen Arendt}

Published in:

2001 IEEE International Ultrasonic Symposium Proceedings

Link to article, DOI:

10.1109/ULTSYM.2001.991982

Publication date:

2001

Document Version

Publisher's PDF, also known as Version of record

Link back to DTU Orbit

Citation (APA):

Schlaikjer, M., \& Jensen, J. A. (2001). Joint probability discrimination between stationary tissue and blood velocity signals. In 2001 IEEE International Ultrasonic Symposium Proceedings (Vol. 1-2, pp. 1397-1400). IEEE. I E E E International Ultrasonics Symposium. Proceedings https://doi.org/10.1109/ULTSYM.2001.991982

\section{General rights}

Copyright and moral rights for the publications made accessible in the public portal are retained by the authors and/or other copyright owners and it is a condition of accessing publications that users recognise and abide by the legal requirements associated with these rights.

- Users may download and print one copy of any publication from the public portal for the purpose of private study or research.

- You may not further distribute the material or use it for any profit-making activity or commercial gain

- You may freely distribute the URL identifying the publication in the public portal 


\title{
Joint probability discrimination between stationary tissue and blood velocity signals
}

\author{
Malene Schlaikjer and Jørgen Arendt Jensen \\ Center for Fast Ultrasound Imaging, Ørsted•DTU, Bldg. 348, \\ Technical University of Denmark, DK-2800 Kgs. Lyngby, Denmark
}

\section{Abstract}

In CFM-mode the blood velocity estimates are overlaid onto the B-mode image. The velocity estimation gives non-zero velocity estimates in both the surrounding tissue and the vessels. A discrimination algorithm is needed to determine, which estimates represent blood flow and should be displayed. This study presents a new statistical discriminator. Investigation of the RF-signals reveals that features can be derived that distinguish the segments of the signal, which do an do not carry information on the blood flow. In this study 4 features have been determined: a) the energy content in the segments before and after echo-canceling, and b) the amplitude variations between samples in consecutive RF-signals before and after echo-canceling. The statistical discriminator was obtained by computing the probability density functions (PDF's) for each feature through histogram analysis of data. The discrimination is performed by determining the joint probability of the features for the segment under investigation and choosing the segment type that is most likely.

The method was tested on simulated data resembling RFsignals from the carotid artery. Discriminators using a combination of one or more of the features have been evaluated. The best performance is obtained, when the discriminator employs the energy content before echo-canceling and the amplitude variations after echo-canceling. A success rate on discrimination of $95 \%$ is obtained on both segment types. The discriminator has been compared to a previously employed method, which uses the amplitude level in the envelopedetected signal. The performance on the segments, which carry blood velocity information, is improved by a factor of 2.3. The segments, which do not carry blood velocity information, is classified equally well with both methods.

\section{Introduction}

Medical ultrasound is used for visualizing the blood velocity distribution in the cardiovascular system. Several processing steps are performed on the acquired RF-signals before the in- formation is presented to the clinicians. Initially the noise is minimized by a matched filter, and the stationary echoes are reduced through echo-canceling. Then the blood velocity estimator is applied. Velocity estimates are produced along the full temporal extend of the RF-signals. This is regardless of whether the individual parts of the signals represent the interactions with tissue structures or the blood. For stationary signals only noise is left after echo-canceling. Consecutively acquired signals from non-moving tissue structures are therefore not identical, and a non-zero velocity results. As the tissues next to moving organs [1], [2] are also moving, the responses from tissue structures will not be at the same temporal location in consecutive RF-signals. The stationary echo-canceling therefore cannot remove the responses from the tissue structures completely, and a non-zero velocity estimate arises in part of the signal representing interaction with tissue structures. Only the blood velocity information is of interest, so a discrimination must be performed prior to display.

The estimated velocities cannot be distinguished by means of their amplitude level, as the values overlap. Instead other features and more complex discrimination algorithms must be developed. In this study a discriminator based on maximum likelihood theory is developed. The feasibility of deriving a set of features from the acquired RF-signals is investigated. In this study it is the set of RF-signals obtained for blood velocity estimation, which are used in the determination of features. The discrimination is not performed on the individual samples of the signals, rather the RF-signals are split up in a number of segments. The features are derived and the discrimination is performed for each of these. The aim of the discriminator is to determine, if the segment under investigation carry information regarding blood velocity.

Discriminators based on neural networks have been developed by a number of authors. Quite a few of these studies focus on tissue characterization (e.g. cancerous/noncancerous prostate tissue) [3], [4]. Neural networks used for the discrimination of the segments, which carry blood velocity information, have also be considered [2], [5]. 


\section{Statistical discrimination}

The design of a discriminator based on maximum likelihood theory requires knowledge of the probability density functions (PDFs) for the features, when the segments do and do not carry blood velocity information. In the following these two types of segments will be referred to as BV and NBV segments, respectively.

The statistical discriminator then computes measures of how likely a given combination of an outcome of the features are, if they are considered representing a BV or a NBV segment. Estimates of the PDFs can be obtained through a histogram analysis of a representative data set. The probability density of a given outcome of the features will be approximated by the joint probability density:

$$
\begin{aligned}
p_{B V} & =p_{1, B V}\left(X_{1}\right) \cdot p_{2, B V}\left(X_{2}\right) \cdot \ldots \cdot p_{F, B V}\left(X_{F}\right) \\
p_{N B V} & =p_{1, N B V}\left(X_{1}\right) \cdot p_{2, N B V}\left(X_{2}\right) \cdot \ldots \cdot p_{F, N B V}\left(X_{F}\right),
\end{aligned}
$$

where $p_{B V}$ and $p_{N B V}$ are the joint probability densities for the BV and NBV segments, respectively. The $p_{i, B V}$ and $p_{i, N B V}$ are the PDFs for the NBV and the BV segments for feature $i$. The $X_{i}$ represent an observed value of feature $i$. This definition assumes that the individual features are independent. This is not the case for the features derived in this study, and the joint probability will be lower than the true probability of the outcome of the features. This decreases the discrimination ability, but this approach is used to ease the implementation with respect to storing the PDFs. The discrimination is performed by computing $p_{B V}$ and $p_{N B V}$. The maximum value of the two joint probability densities determines the type of the segment. The statistical basis for the statistical discriminator hereby has been defined. The next step is to determine a set features, which distinguish the NBV and the BV segments. A total of 4 features are suggested based on investigations of the acquired RF-signals.

The amplitude level of the backscattered signal from blood is $10-40 \mathrm{~dB}$ lower than that from the surrounding tissue. The amplitude level in the acquired RF-signals from arteries will vary, as the pulse has interacted with tissue and blood structures, respectively as shown in Fig. 1. By splitting the signal up in a number of segments, the energy content can be used as a feature to distinguish:

$$
F_{1}(m)=\frac{1}{\sigma} \sum_{l=1}^{N_{l}} \sum_{k=1}^{N_{s}} r\left(l, k+m N_{s}\right)^{2},
$$

where $\sigma$ is a normalization factor equal to the standard deviation of an RF-signal (averaged over a set of RF-signals). The RF-signal is represented by $r, l$ is the line number, and $m$ indicates the segment number. The variables $N_{s}$ and $N_{l}$ represent the number of samples in a segment and the number of consecutive RF-signals, respectively. After echo-canceling a large portion of the tissue signal has been removed. The amplitude level in the region containing the interaction with the

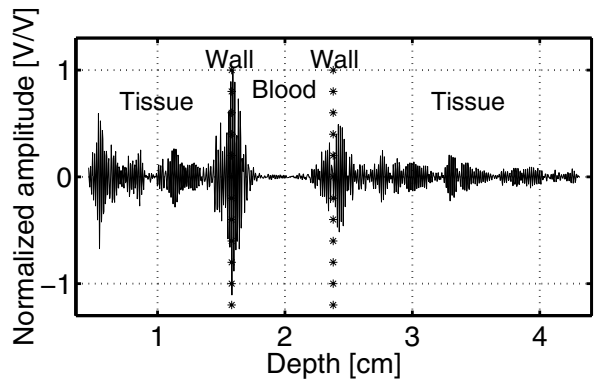

Figure 1: RF-signal acquired at the carotid artery.

blood will often have a higher amplitude than in the tissue region. This property is used as the second feature:

$$
F_{2}(m)=\frac{1}{\sigma} \sum_{l=1}^{N_{l}-1} \sum_{k=1}^{N_{s}} r_{e c h o}\left(l, k+m N_{s}\right)^{2},
$$

where $r_{\text {echo }}$ represents the echo-canceled data. The reflected signals from structures, which are moving, will be temporally shifted in two consecutive RF-signals. The standard deviation on a set of samples from consecutive signals at a given temporal position will therefore be non-zero. The motion of the tissue is most often much lower than the motion of the blood [1], [2], so the level of variation can be used as a feature:

$$
F_{3}(m)=\frac{1}{\sigma} \sum_{k=1}^{N_{s}} \sqrt{\frac{1}{N_{l}-1} \sum_{l=1}^{N_{l}}\left(r\left(l, k+m N_{s}\right)-\mu_{k+m N_{s}}\right)^{2}},
$$

where $\mu_{k+m N_{s}}$ is the mean value of the set of samples from the set of consecutive lines at the temporal location $k+m N_{s}$ (in samples). The variation is also present in the echo-canceled signals, and it is used as feature 4 :

$$
F_{4}(m)=\frac{1}{\sigma} \sum_{k=1}^{N_{s}} \sqrt{\frac{1}{N_{l}-2} \sum_{l=1}^{N_{l}-1}\left(r_{e c h o}\left(l, k+m N_{s}\right)-\mu_{e c h o, k+m N_{s}}\right)^{2}}
$$

The development of the statistical discriminator was based on simulated data, which resemble RF-data for CFM imaging obtained at the carotid artery. These data are well-defined with respect to the exact location and extend of the tissue and vessels (unlike in-vivo data). The simulation program Field II [6], which can handle any transducer array, excitation waveform, and focusing and apodization scheme, was employed. A scan setup matching a B-K Medical A/S 3535 scanner connected to a 128 elements linear array was implemented. A 6 cycle pulse with a $5 \mathrm{MHz}$ center frequency is emitted. The vessel diameter is $6 \mathrm{~mm}$, and it lies at an angle of $35^{\circ}$. Three cardiac cycles were simulated. Tissue motion due to pulsation of the vessel walls [1], [2] was included. Womersleys model 
[7] was used to model the blood motion. Eight RF-signals were acquired for each line in the CFM image. A time gain compensation is applied to counteract the attenuation.

A histogram analysis of the data for a SNR of $10 \mathrm{~dB}$ gives the PDFs, which are plotted in Fig. 2. The features span a normalized range from 0 to 15 , and this region was split up in 76 intervals. As the unit of the RF-signals is Volt $(V)$, the features have the units $V^{2} / V$ and $V / V$. The PDFs reveal that the features do distinguish the BV and NBV segments in most cases. The overlap is due to noise, tissue motion, and overlap between blood and tissue signals at the vessel wall. The PDFs vary as the SNR is varied. The position of the PDFs are shifted slightly up or down, and the level of overlap varies. Still it is feasible to use these features for discrimination.

\section{Results}

The discriminator in (1) uses all four features, but any combination can be used. The performance for the 15 combinations of the 4 features has been investigated. A discriminator using $F_{1}$ and $F_{4}$ gives the best performance. The subjective for choosing the best discriminator was twofold: 1) the number of correctly discriminated NBV and BV segments must be as high as possible, and 2) the misclassified segments should not be systematic (e.g. show up in the same phase of the cardiac cycle). The number of correctly discriminated segments are listed in Table 1. The success rate is above $95 \%$ for a SNR above $0 \mathrm{~dB}$, and most BV segments are classified correctly. The majority of the misclassified segments are located at the transition zone between the vessel and the tissue regions. The extend of the vessel is both over and under estimated. Figure 3 shows a typical outcome of the discrimination. As a

\begin{tabular}{|cl||c|c|c|c|}
\hline & SNR & 0 & 10 & 20 & 30 \\
\hline \hline Stat & $N_{N B V}$ & 91.0 & 96.5 & 96.9 & 96.4 \\
& $N_{B V}$ & 80.5 & 95.1 & 98.1 & 98.9 \\
\hline Amp & $N_{N B V}$ & 97.8 & 97.1 & 97.0 & 97.0 \\
& $N_{B V}$ & 42.6 & 42.1 & 42.3 & 42.3 \\
\hline
\end{tabular}

Table 1: The number of correctly discriminated BV and NBV segments (in \%) with the statistical (Stat) and the amplitude (Amp) discriminator for a range of SNRs.

reference a discrimination based on a comparison of the average amplitude $A(m)$ of the envelope detected data $r_{e n v}$ to a threshold value is used. The average amplitude is defined as:

$$
A(m)=\frac{1}{\sigma} \sum_{l=1}^{N_{l}} \sum_{k=1}^{N_{s}} r_{e n v}\left(l, k+m N_{s}\right) .
$$

The threshold function is designed as a step function, which decreases as a function of depth. No time gain compensation
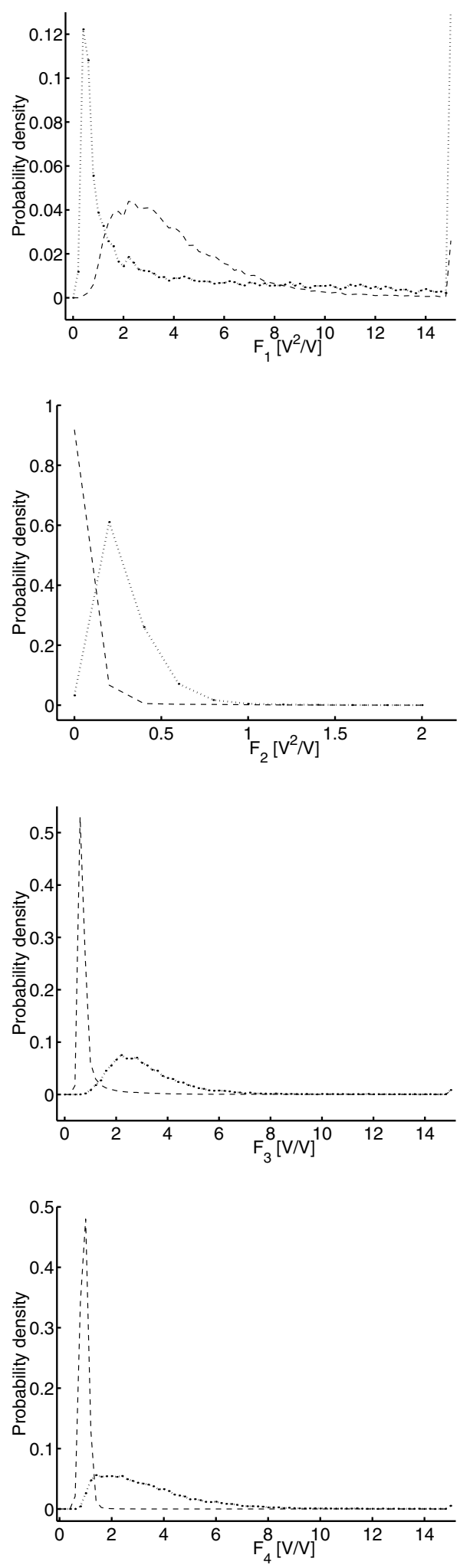

Figure 2: The probability density functions (PDF) of feature 1-4 for a SNR of $10 \mathrm{~dB}$ for the NBV (dashed) and BV (dotted) segments. 
(a)
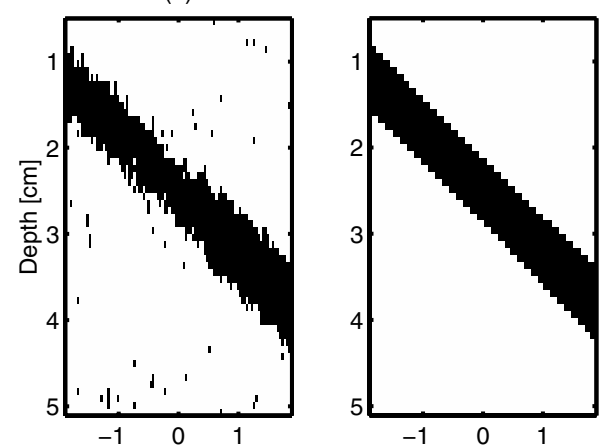

(b)
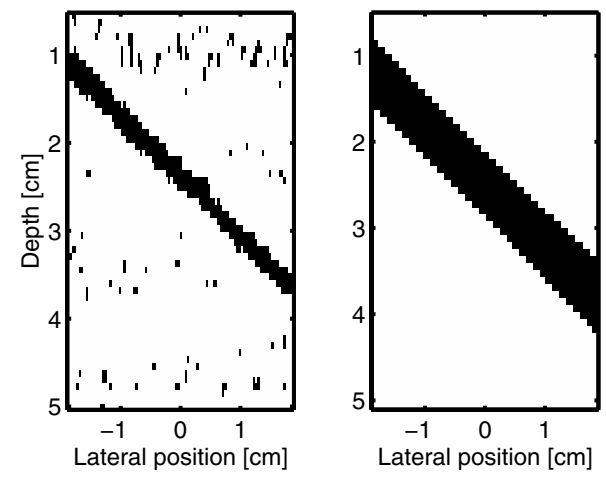

Figure 3: Performance of the statistical (a) and amplitude discriminator (b) for a SNR of $10 \mathrm{~dB}$. The true discrimination is shown in the right column.

of the RF-signals is applied in this case. The performance of the amplitude and statistical discriminators has been compared, and the results are listed in Table 1 and shown in Fig. 3. The statistical discriminator outperforms the amplitude discriminator by a factor of 1.9 and 2.3 for the BV segments, when the SNR is equal to and above $0 \mathrm{~dB}$, respectively. This difference in the ability to discriminate the BV segments is shown in the plots. The amplitude discriminators only works well at the center of the vessel. This is due to the overlap of signals from blood and tissue at the level of the vessel walls. The performance on the NBV segments is almost identical for the two methods.

\section{Conclusion}

The introduction of more features and more advanced algorithms improved the basis for discrimination of segments that carry blood velocity information. The new statistical discriminator comes close to discriminating all the segments correctly and outperforms the simple amplitude discriminator. Still, more features should be considered. The segment types of temporal and spatial neighbor segments may contain valuable information. Another problem is that the definition of the features contain a normalization constant, which depends on the absolute amplitude level in the RF-signals. The PDFs thereby get to be dependent on the medium of propagation, which is inconvenient. The feasibility of using features, which represent the ratio of $e . g$. the energy before and after echo-canceling should be considered and evaluated in the future.

\section{Acknowledgement}

This work was supported by grant 9700883, 9700563, and 5013-00-0025 from the Danish Science Foundation, grant 980018-311 from the Technical University of Denmark, Denmark, and by B-K Medical A/S, Gentofte, Denmark.

\section{References}

[1] M. Schlaikjer, S. Torp-Pedersen, J. A. Jensen, and P. F. Stetson. Tissue motion in blood velocity estimation and its simulation. In Proc. IEEE Ultrason. Symp., pages 1495-1499, 1998.

[2] M. Schlaikjer. Development and characterization of algorithms for estimation of blood velocity with ultrasound. $\mathrm{PhD}$ thesis, Ørsted•DTU, Technical University of Denmark, Kgs. Lyngby, Denmark, 2001.

[3] E. J. Feleppa, W. R. Fair, T. Liu, A. Kalisz, K. C. Balaji, C. R. Porter, H. Tsai, V. Reuter, W. Gnadt, and M. J. Miltner. Three-Dimensional Ultrasound Analyses of the Prostate. Mol Urol, 4:133-139, 2000.

[4] L. Kahl, R. Orglmeister, and K. J. G. Schmailzl. A Neural Network Based Classifier for Ultrasonic Raw Data of Myocardium. In Proc. IEEE Ultrason. Symp., pages 1173-1176, 1997.

[5] M. Schlaikjer and J. A. Jensen. Neural network for RFsignal decomposition in ultrasound : a pilot study. In Proc. of Neural Computation, pages 628-633, 2000.

[6] J. A. Jensen. Field: A program for simulating ultrasound systems. Med. Biol. Eng. Comp., 10th Nordic-Baltic Conference on Biomedical Imaging, Vol. 4, Supplement 1, Part 1:351-353, 1996b.

[7] W. W. Nichols and M. F. O'Rourke. McDonald's Blood Flow in Arteries, Theoretical, Experimental and Clinical Principles. Lea \& Febiger, Philadelphia, 1990. 